\title{
Reverse time migration of tunnel seismic reflected data
}

\author{
Xu Chang, Yike Liu \\ Institute of Geology and Geophysics, Chinese Academy of Sciences \\ changxu@mail.igcas.ac.cn
}

\begin{abstract}
Reverse time migration method with forward modelling zero-offset seismic data is applied to the prediction ahead of tunnel face. The numerical model with soft rock mass zone and fault zone are used to test the reverse time migration for locating the positions of fault zone ahead of tunnel face. The numerical simulation results have proven that reverse time migration with zero-offset data is exactly able to image the fault zone in un-excavation ahead of tunnel.
\end{abstract}

\section{Introduction}

Geophysics surveys have been widely used in determining the subsurface structure and its physical properties. Recently, the research target of geophysical survey is not only to the earth resources exploration but also to environment and civil engineering. City development and urbanization, for example subway, highway, harbor and airport construction, bring in the interests of development and effective utilization of underground. Downtown and poor ground investigating, and the process and safety management in civil engineering construction need geophysical technique to support. Seismic survey is one of the geophysical methods to understand underground.

Predicting ahead of tunnel face is implemented for the understanding to the forward geological structure, such as faults or fractured zone during the excavation of tunnel. Looking ahead of tunnel face is a challenge technique. Many successful research results of numerical methods and tunnel sites have been published until now. Equi-traveltime planes method for looking ahead of tunnel face was provided by Kyoto University (Ashida, et al., 1998). The method indicates that the concept of equi-travaltime planes shows successful result in predicting ahead of tunnel face by a use of new arrangement way of sources and receivers. Tomography method was also proposed to predict un-excavation area. Estimating the distribution of excavation disturbed zone based on the combining acoustic and resistivity tomography was firstly used in the tunnel and cavern site (Suzuki, et al., 2003). Tunnel seismic prediction and horizontal seismic profiling are the current techniques in common use in tunnel construction site. In recent years, seismic migration is an attentive method for predicting ahead of tunnel face. Kirchhoff depth migration and some of its improved method have been published (Goertz, et al., 2003). These methods still have some problems need to be solved. For example the predicting delays the construction speed, and the observation 
geometry is hard to arrange. The prediction method without troublesome procedure is really expected by the engineers in the excavation site. In this paper, we use zero-offset seismic data and reverse time migration for imaging un-excavation tunnel area.

\section{Reverse time migration method}

Reverse time migration method based on wave equation finite difference approximation has been published and used in seismic oil exploration (Baysal, et al., 1983). Reverse time migration is according to the reversibility of finite difference algorithm in time step. This migration method is a reversion of wave equation modelling. The imaging result is in the depth domain and the method is effective for imaging the slope stratum with big angle. The study in this paper is using acoustic wave equation to do numerical simulation.

$$
\frac{\partial^{2} U}{\partial x^{2}}+\frac{\partial^{2} U}{\partial z^{2}}=\frac{1}{V^{2}(x, z)} \frac{\partial^{2} U}{\partial t^{2}}
$$

Equation (1) is the two dimensional scalar acoustic wave equation. $U$ is the press, $x$ and $z$ indicate the coordinates of spatial domain, $t$ is the acoustic travel time, and $V$ is the seismic velocity of the underground medium. The discrete formula of equation (1) is shown by equation (2).

$$
\begin{aligned}
U_{i, j}^{k+1} & =2\left(1-\alpha^{2}-\beta^{2}\right) U_{i, j}^{k} \\
& +\alpha^{2}\left(U_{i+1, j}^{k}+U_{i-1, j}^{k}\right) \\
& +\beta^{2}\left(U_{i, j+1}^{k}+U_{i, j-1}^{k}\right)-U_{i, j}^{k-1}
\end{aligned}
$$

where

$$
\begin{aligned}
& \alpha=V \cdot \Delta t / \Delta x \\
& \beta=V \cdot \Delta t / \Delta z
\end{aligned}
$$

and $i, j$, and $k$ are the subscripts of $\mathrm{x}, \mathrm{z}$, and $\mathrm{t}$, respectively, $\Delta \mathrm{x}, \Delta \mathrm{z}$, and $\Delta \mathrm{t}$ are the interval of the $x, z$, and $t$, respectively.

The initial condition is the seismic source in forward modelling, whereas the initial condition is the seismic records in reverse time migration. The calculation is from zero to the maximum number of record samples by time step in the forward modelling, whereas the calculation is from the maximum number of record samples to zero time by time step. The migration imaging condition is $t=0$. Reverse time migration calculation is executed in depth domain, and output a depth domain image. In order to do geological prediction ahead of tunnel face, a reverse time migration is proposed in our study. The input data is zero-offset record, and the output data is the geological situation of un-excavation tunnel area.

\section{Zero-offset data in tunnel}

A simple seismic observing way without troublesome procedure is important during the excavation job. So, we suppose that only one seismic trace is collected when an acquisition is implemented during the detecting process for tunnel faces. At first, however, we need a 
number of zero-offset records, such as 48 records, to carry out the reverse time migration. These records were obtained by moving the shot and geophone pair sequentially until the number that we need to migrate. After the first migration we are moving forward one interval space with the progress of excavation work. A new one record was collected and old one record was deleted. All of the subsequent migration prediction uses 48 records, and it can be finished in a real time. Fig.1 shows a geological model with some soft rock zones and the early situation of progress of excavation. Seismic receivers are placed on the top and bottom of the tunnel. The sources have the same position as the receivers.

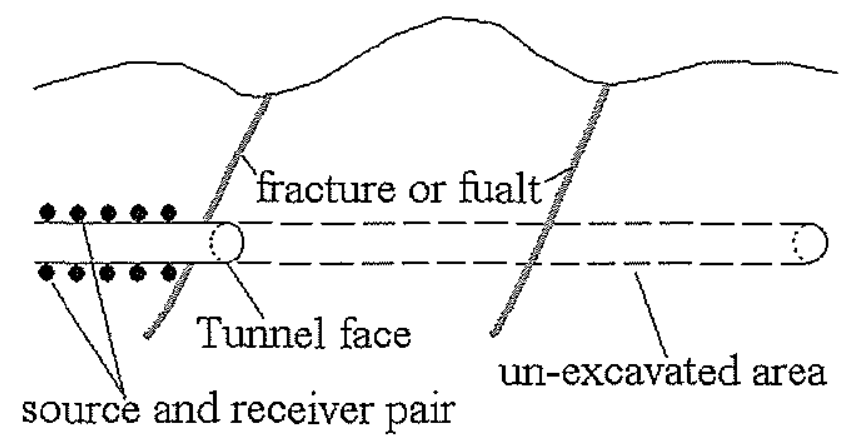

Fig.1 A geological model with fractures or faults. The source and receiver pair is arranged on the top and bottom of the tunnel.

\section{Numerical imaging result for prediction}

In the numerical calculation, two types of seismic source were used. One is the explode surface source, and the other is point source. Fig.2 shows the model with a soft rock zone, and its reverse time migration image by using explode surface source. Two faults generated by the reverse time migration with different directions were shown in Fig.2. The fault on the above of the tunnel is the artifact and the fault in the bottom of the tunnel is true image.
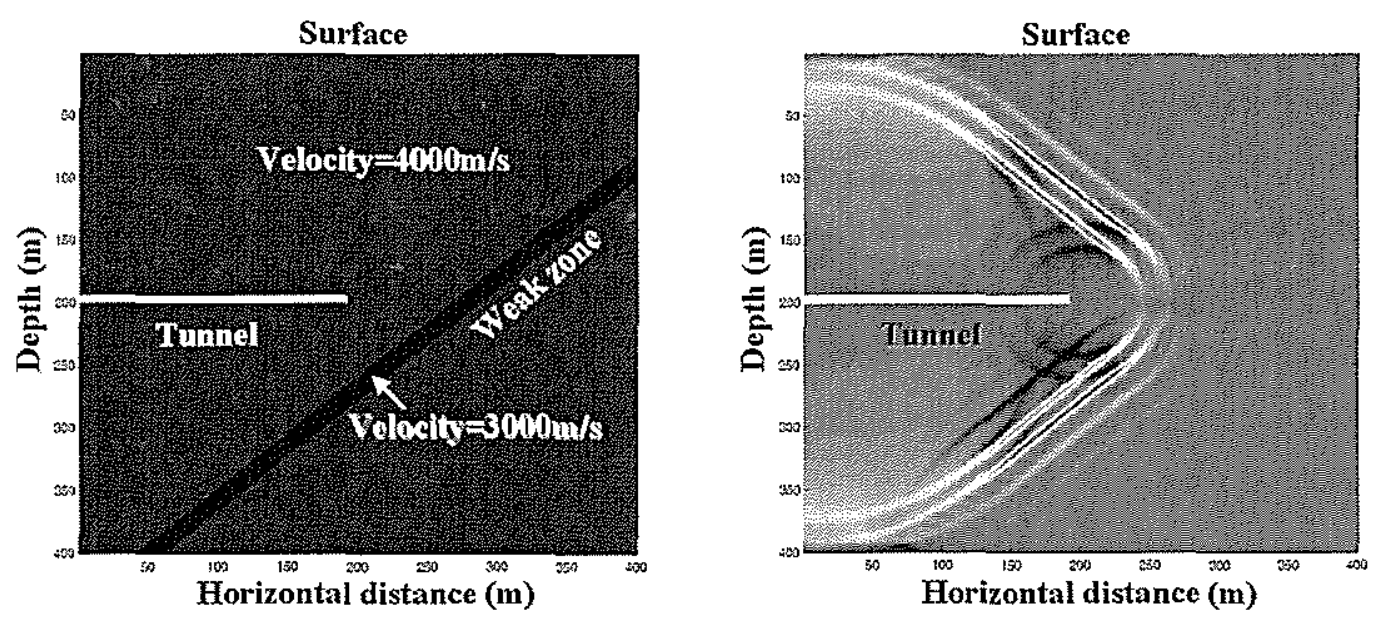

Fig.2 One fault model (left) and its reverse time migration image (right).

The migration artifact in conventional reverse time migration is impossible to remove. This 
result indicates the significance of interpretation.
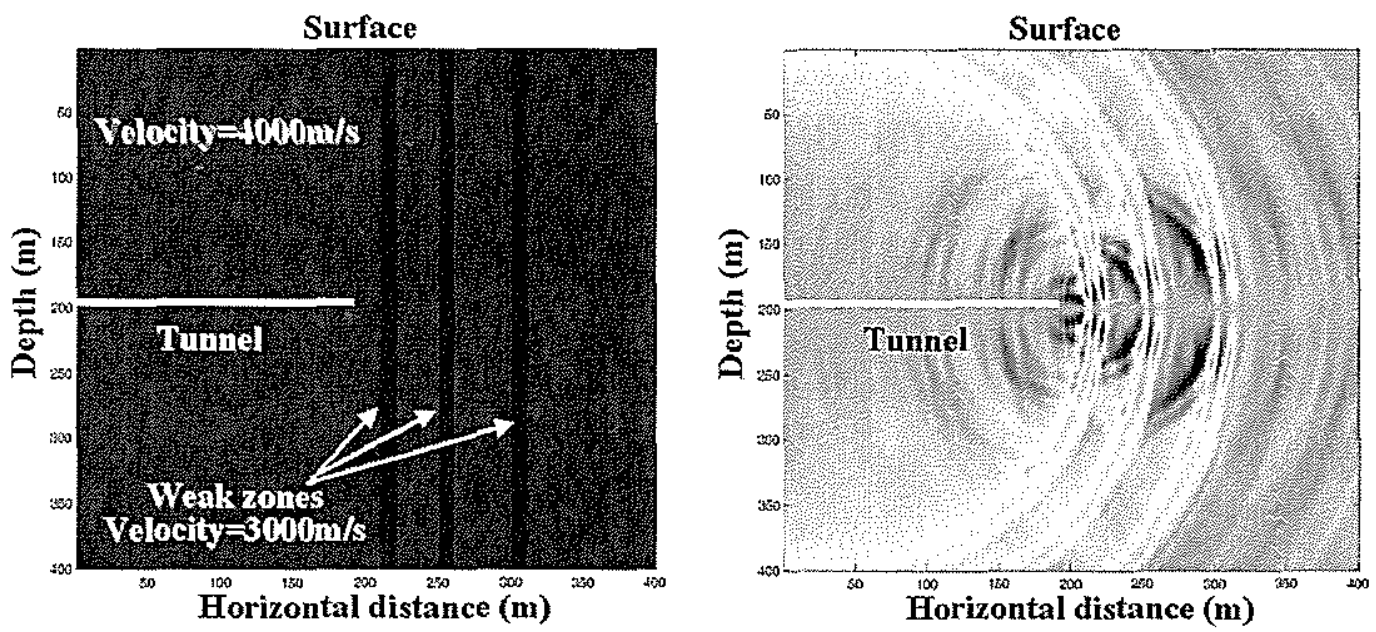

Fig. 3 Three parallel fractures model (left) and its reverse time migration image (right).

Fig. 3 shows a model with three parallel fractures and its migrated image. The result indicates that the image will be correct if explode surface source is used in zero-offset reverse time migration. The explode surface source is too perfect to realize, and point source (shown in Power Point) should be introduced into the numerical simulation.

\section{Discussions}

Zero-offset reverse time migration method is applied to predict ahead of tunnel face. The numerical results show the method is successful by using explode surface source. However, the artifact remove technique and migration calculation by using point source condition are still challenging work need to be studied.

\section{Acknowledgement}

This research is supported by National Natural Sciences Foundation of China, project number is 40235055 .

\section{References}

Ashida Yuzuru, Matsuoka Toshifumi, and Watanabe Toshiki, Imaging algorithn for looking ahead prediction of near subsurface data, 1998, Proceedings of the 4th SEGJ International Symposium, Tokyo, Japan, 129-134.

Suzuki Koichi, Nakata Eiji, Minami Masayuki, Hibino Etsuhisa, Tani Tomonori, Sakakibara Jyunichi, and Yamada Naoyuki, Estimation of excavation disturbed zone in rock around caverns using resistivity tomography and acoustic tomography, 2003, Proceedings of the 6th SEGJ International Symposium, Tokyo, Japan, 414-417.

Goertz A., Muller Ch., Buske S., Lueth S., Fresnel-volume multicomponent migration, 2003, EAGE 65th Conference and Exhibition, Stavanger, Norway, Z-99.

Baysal E., Kosloff D. D., and Sherwood J. W. C., reverse time migration, Geophysics, 1983, $48(11), 1514-1524$. 